\title{
SLIT SCATTERING EFFECTS IN A WELL ALIGNED PEPPER POT
}

\author{
John G. Power, ANL, Argonne, IL, 60439
}

\begin{abstract}
A pepper pot is a device used to measure a medium energy $(<20 \mathrm{MeV})$ electron beam's transverse emittance by sampling its transverse phase space. This is accomplished by blocking most of the incident electron beam, while allowing small 'beamlets' to pass through openings in a mask. The accuracy of the transverse emittance measured by a pepper pot is limited by several factors including, electrons leaking through the solid region of the mask, the imaging system resolution and dynamic range, scattering, etc. While the noise contributions from the prior quantities can be easily estimated, scattering effects have previously been neglected due to the difficulty in estimating the effect. In this paper, EGS4 simulations are presented to determine the affect of scattering on emittance measurements for an $8 \mathrm{MeV}$ electron beam.
\end{abstract}

\section{INTRODUCTION}

In general, it is difficult to measure the transverse beam emittance directly out of an RF photocathode gun because the emittance is small, the charge is high, and the energy is low, so that the beam is space charge dominated. For this reason, the emittance is usually measured after the RF photoinjector - defined here to be an RF photocathode gun and at least one linac tank - so that the energy of the electron beam is higher, typically greater then $20 \mathrm{MeV}$. At higher energy, and hence lower space charge, there are several reliable methods for measuring the emittance: (1) The Quad Scan; (2) The Three Screen Method; and (3) OTR-based measurements. However, these three methods cannot easily be applied for the case considered here.

In the traditional quad-scan method, the space charge force is neglected and an exact analytic expression is used to track the beam envelope evolution. However, if the beam is space charge dominated this method fails since the beam envelope evolves under the influence of both emittance and space charge [2, 3]. While the traditional method has been modified to include space charge $[2,4]$, this method is still not ideal since it is not a single-shot measurement - something that is important for photoelectron beams due to the shot-to-shot fluctuation of the laser. And while the three screen method is limited by the same space charge considerations as the traditional quad scan, OTR-based techniques do not suffer from space charge limitations. However, since the angular distribution of the OTR light at the beam waist depends on both the divergence and the angle energy correlation induced by the quad focusing of the photoelectron beam. Therefore, this technique can be limited by a large energy spread (1\%) of the photoinjector. Recently, a promising model has been developed [5] using a technique related to OTR, based on optical diffraction radiation (ODR-OTR interference) that may overcome even this last limitation.

The pepper pot (and emittance slit) based method does not suffer from the above limitations. However, concerns have been raised that the electron beam may undergo slit scattering as it passes through the pepper pot. The slit scattering process and the degree to which it affects the measured emittance is described in the remainder of the paper.

\section{SLIT SCATTERING}

The function of the pepper pot collimator is to stop or scatter away the fraction of the incident electron beam that is intercepted by the plate while letting the nonintercepted fraction of the incident beam pass through the hole. (1.h.s. of Fig. 1). In the case of an ideal pepper pot with one round hole, the non-intercepted beam signal (beamlet) emerges from the hole as a cylinder of charge while the intercepted beam is either stopped. In the real case, however, some of the intercepted beam will enter the plate, undergo a series of interactions, and then reemerge from it as a source of noise which may affect the measured profile of the non-intercepted signal thus limiting the accuracy of the emittance measurement. In this paper we will examine how this noise affects the measurement of the signal. To keep matters simple, a pepper pot with a single hole is studied.

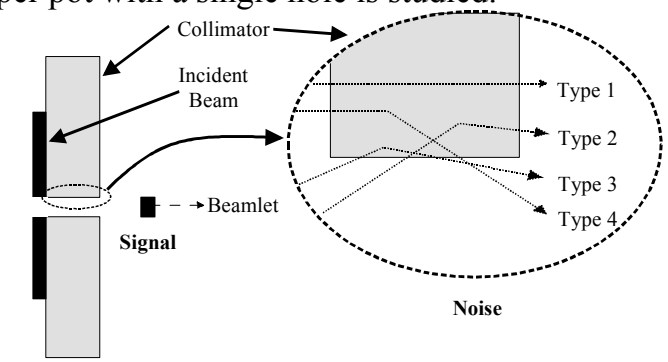

Figure 1: The incident beam strikes a collimator with one hole. The non-intercepted beam is the signal (beamlet) and some of the intercepted beam produces noise (Types $1-4)$ in the measurement.

For clarity, we divide the noise into four components as shown on the r.h.s. of Figure 1. The first component of the noise (Type 1) is the intercepted beam that enters the front face and exits the back face of the collimator and is termed the leakage component. The distribution of the leakage component can be readily estimated from the well known atomic and nuclear properties of the collimator material. The angular and spatial distributions of the leakage component can be obtained from the standard multiple Coulomb scattering formula [6] while the fraction of the intercepted beam that is stopped in the material can be estimated with the stopping distance from the minimum ionizing energy, typically $1-2 \mathrm{MeV} \mathrm{cm}^{-2} \mathrm{~g}$. 
The other three noise components, taken together, make up the slit scattered component. Types 2 and 3 particles start out inside the hole but end up striking the plate due to their initial trajectories, while Type 4 particles initially strike the front face of the collimator but are scattered back into the hole. Unlike for the leakage component, there are no simple formulas to guide one in estimating the slit scattered contribution to the noise.

\section{SIMULATION}

The method used to simulate the problem of an electron beam incident on a single collimating hole was as follows: (1) generate an incident beam at the beginning of the pepper pot; (2) transport the incident beam to the end of the pepper pot with an electromagnetic shower code; (3) transport all particles through a drift to the location of the screen; and (4) analyze the output distribution. A user written routine, using the SDDS Toolkit [7], was used to generate the monochromatic incident beam of zero length and the toolkit's Gaussian random number generator was used to populate the angular profiles. The interaction of the incident electron beam with the collimator was modeled with Shower [7] which is a SDDS-compliant interface program to the Monte Carlo electron-photon transport program EGS4 [8]. The propagation of the beam in the drift and the post-processing analysis were done with user written Matlab files.

\section{Electron Beam and Pepper Pot Parameters}

This study of slit scattering was motivated by need to measure the emittance of the new 1.5 cell RF photocathode gun at the Argonne Wakefield Accelerator (AWA). This gun was recently RF conditioned to 80 $\mathrm{MV} / \mathrm{m}$, on the cathode, corresponding to $12 \mathrm{MW}$ of input power. PARMELA simulations show that with $80 \mathrm{MV} / \mathrm{m}$ and a flat-flat laser pulse profile the gun will produce a photoelectron beam with $\mathrm{E}=7.5 \mathrm{MeV}, \mathrm{Q}=40 \mathrm{nC}, \varepsilon_{\mathrm{n}}=$ $66 \mathrm{~mm} \mathrm{mrad}, \sigma_{\mathrm{Z}}=1.1 \mathrm{~mm}(3.7 \mathrm{psec})$, and $\Delta \mathrm{E} / \mathrm{E}=2 \%$. At an energy of $7.5 \mathrm{MeV}$ we have $\gamma=15.6$ so that the geometrical emittance $\varepsilon \sim 4.2 \mathrm{~mm}$ mrad. Initially, however, the AWA laser profile will be either Gaussian Gaussian or longitudinally Gaussian - transversely flat so that $\varepsilon_{\mathrm{n}} \sim 100 \mathrm{~mm} \mathrm{mrad}$ is a better estimate which means that $\varepsilon \sim 6.4 \mathrm{~mm}$ mrad. The plan is to measure the emittance about $50 \mathrm{~cm}$ after emittance compensating solenoid where we have a round beam near a waist with $\sigma_{x, y} \sim 5 \mathrm{~mm}$ so that the divergence is approximately $\sigma_{x, y}^{\prime} \sim 1.5 \mathrm{mrad}$. These parameters will be used as basis for the incident beam during the simulation.

Based on the above assumptions about the photoelectron beam, a pepper pot was designed for measuring the emittance. Since slit scattering can be understood by just considering a single hole, the overall hole pattern will not be describe but, instead, discussion will be confined to just a plate with a single hole [Fig. 2].

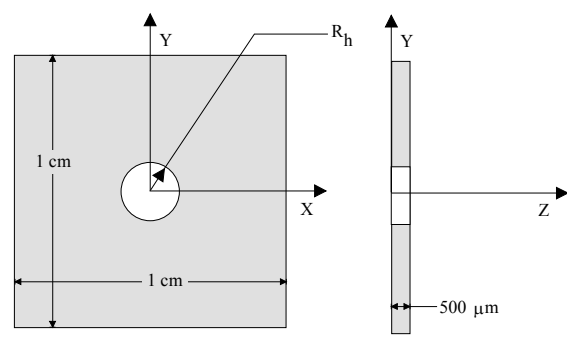

Figure 2: The single hole pepper pot collimator. (Not to scale.)

The parameters used for the pepper pot in this study were a square plate of tungsten $(1 \mathrm{~cm} \mathrm{x} 1 \mathrm{~cm}$ and $500 \mu \mathrm{m}$ thick) with a hole of radius $R_{h} \mu \mathrm{m}$ in the center. Using the radiation length, $\mathrm{X}_{0}=3.5 \mathrm{~mm}$ a and density $\rho=19.3$ $\mathrm{g} \mathrm{cm}^{-3}$ of tungsten, we calculate the multiple coulomb scattering of the $7.5 \mathrm{MeV}$ beam from the $500 \mu \mathrm{m}$ thick plate to be $\theta_{\mathrm{mcs}}=635 \mathrm{mrad}$. The geometry used for this pepper pot (Fig. 2) defines the beginning of the tungsten plate to be at $\mathrm{z}=0$, the exit of the tungsten plate at $\mathrm{z}=$ $500 \mu \mathrm{m}$, the profile monitor at $\mathrm{z}=\mathrm{L}$, and the hole radius of $R_{h}$.

\section{RESULTS}

Shower was used to simulate an initial distribution of electrons incident on the pepper pot described above. This initial distribution is a round, uniformly populated electron beam of radius $R_{b}=500 \mu \mathrm{m}$, with a Gaussian angular distribution of $\sigma_{x, y}^{\prime}=1.5 \mathrm{mrad}$.

As an example, the total output distribution of particles (electrons, photon, and positrons) located at the back face of a pepper pot with $R_{h}=100 \mu \mathrm{m}$ is shown in figure 3 . Looking at the profile from this point of view, it is not possible to pick the signal $(100 \mu \mathrm{m}$ spot $)$ out of the noise $(\leq 500 \mu \mathrm{m}$ spot). However, by plotting the intensity of the particle distribution along a slice through the middle of the profile (figure 3), a small signal can be observed. In figure 4 , this intensity along the center of the slice is shown at four different locations behind the pepper pot (where $\mathrm{z}=0$ is the back face of the pepper pot). The first feature to notice is that there is small, sharply peaked signal sitting on the center of a hill of noise. At the exit of the plate $(z=0$ in figure 4$)$ the noise hill is rather concentrated $\left(\sigma_{\text {noise }} \sim 500 \mu \mathrm{m}\right)$ but quickly spreads out due to its large angular divergence so that by the time it has traveled just $5 \mathrm{~mm}$ away, the background noise is just a low level, wide hill.

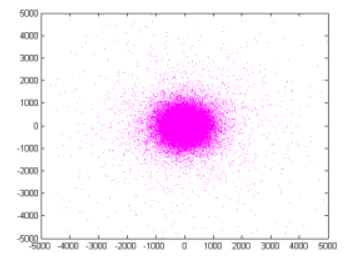

Figure 3: Image of the electrons, photons, and positrons that emerge at the back of the tungsten plate. 


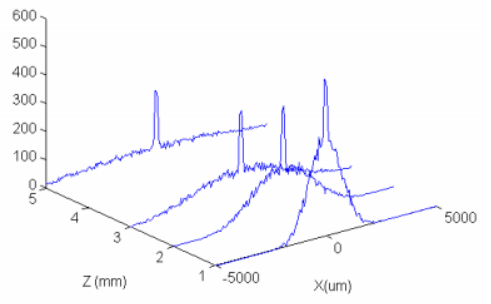

Figure 4: The evolution of the distribution of particles after having passed through the one-hole pepper pot of Fig 2 as a function of distance after the pepper pot.

\section{Calculating the Divergence}

Since the main purpose of these simulations was to see how the slit scattered particles affected the measurement of the signal particles at the downstream screen, the next step was to analyze the downstream spot to see if we can extract the initial divergence of the input beam. The procedure used to do this is described next.

For a particular hole radius, $R_{h}$, the output distribution was propagated to a downstream screen a distance $\mathrm{L}_{\text {screen }}$ $=50 \mathrm{~cm}$ away. Since the input divergence was manually set to have a Gaussian distribution, the spot on the downstream screen was first projected along the $\mathrm{x}$ axis (not sliced) and fit to a Gaussian. From this projection, the 1-sigma beam width of the spot was extracted, which, for the case of a Gaussian, is also equal to the standard deviation of the distribution. The width of the spot due to the divergence of the beamlet is added in quadrature with the r.m.s. width of the pepper pot hole to give the width of the spot at the screen. From this, the divergence is calculated according to,

$$
\sigma^{\prime}=\frac{1}{L_{\text {screen }}} \sqrt{\sigma_{\text {screen }}^{2}-\left(\frac{R_{h}}{2}\right)^{2}}
$$

where $\sigma_{\text {screen }}$ is the spot size at the screen. (Note that while the r.m.s. width of a $1 \mathrm{D}$ slit is $R_{h} / \sqrt{3}$, we are using the r.m.s. width of a projected hole.)

As a typical example, the intensity distribution of the projected beamlet spot for the case of $R_{h}=50 \mu \mathrm{m}$ and $\mathrm{L}_{\text {screen }}=50 \mathrm{~cm}$ is shown in figure 5 . From the fit to this spot the width of the beam at the screen was found to be $\sigma_{\text {screen }}=738 \mu \mathrm{m}$. Using Eqn. 1, the divergence was calculated to be $\sigma^{\prime}=1.51 \mathrm{mrad}$, in very close agreement with the divergence of the initial beam distribution.

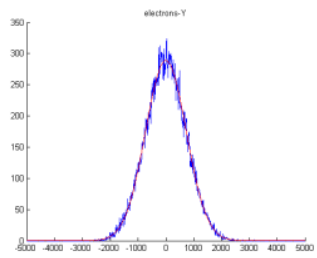

Figure 5: The intensity distribution of the projected beamlet at the screen located $50 \mathrm{~cm}$ downstream of the pepper pot.
Table 1: Calculated divergence from the fit as a function of the aspect ratio of the hole for the case of a pepper thickness of $\mathrm{W}=500 \mu \mathrm{m}$.

\begin{tabular}{|c|c|c|}
\hline $\begin{array}{c}\mathrm{R}_{\mathrm{h}} \\
(\mu \mathrm{m})\end{array}$ & $\begin{array}{c}\text { Aspect Ratio }= \\
\mathrm{R}_{\mathrm{h}} / \mathrm{W}(\mathrm{mrad})\end{array}$ & $\begin{array}{c}\text { Divergence } \\
\text { from Fit }\end{array}$ \\
\hline 10 & 20 & 1.49 \\
\hline 20 & 40 & 1.49 \\
\hline 50 & 99.6 & 1.51 \\
\hline 100 & 197 & 1.50 \\
\hline 200 & 381 & 1.51 \\
\hline 500 & 785 & 1.49 \\
\hline
\end{tabular}

To determine if the multiple scattering effects depended on the aspect ratio of the hole, a variety of cases were considered. In all cases considered, the thickness of the tungsten plate was held at $\mathrm{W}=500 \mu \mathrm{m}$, while the hole radius was allowed to vary from $10 \mu \mathrm{m}$ to $500 \mu \mathrm{m}$, thus varying the acceptance angle of the hole ranged from $20 \mathrm{mrad}$ to $785 \mathrm{mrad}$. As shown in table 1, the fitted value of the divergence agrees very closely to the known value of divergence of $1.5 \mathrm{mrad}$ for a wide range of aspect ratios. The small disagreement is due to the statistics from the limited number of macro particles used in the Monte Carlo simulation.

It is worth emphasizing that the simulation results obtained in this section, and shown in figure 3, figure 4, and table 1, include all the sources of flux mentioned in the SLIT SCATTERING section above, i.e. leakage flux (type 1), slit scattered flux (type 2-4) and the beamlet signal. However, from these simulation result only two fluxes are apparent, the signal and leakage particles. It is obvious that the slit scattering effect is very small over the range of parameters examined.

\section{CONCLUSIONS}

EGS4 based simulations show that the effect of slitscattering on an $8 \mathrm{MeV}$ electron beam by a well aligned pepper pot, with various hole aspect ratios, is negligible. Thus, the pepper pot is a highly reliable technique for measuring emittance from a photoinjector. In the future, the study will be carried out over a wider range of energies and for misaligned pepper pots.

\section{REFERENCE}

[1] M. Reiser, Theory and Design of Charged Particle Beams (Wiley, New York, 1994).

[2] J. G. Power, M. E. Conde, Rev. Sci. Inst. 691295 (1998)

[3] S. G. Anderson, et al., Phys. Rev. ST Accel. Beams 5 014201 (2002)

[4] C. Limborg, these proceedings

[5] R. Fiorito, these proceedings

[6] Particle Data Book

[7] see www.aps.anl.gov for SDDS Toolkit \& Shower

[9] see www.slac.stanford.edu/egs/ for EGS4 